University of Nebraska - Lincoln

DigitalCommons@University of Nebraska - Lincoln

Faculty Publications in Computer \& Electronics Electrical \& Computer Engineering, Department Engineering (to 2015)

2007

\title{
Design of a 1.8V 8-bit 500MSPS Folding-Interpolation CMOS A/D Converter with a Folder Averaging Technique
}

\author{
Dongjin Lee \\ Dongguk University \\ Jaewon Song \\ Dongguk University \\ Jongha Shin \\ Dongguk University \\ Sanghoon Hwang \\ Dongguk University \\ Minkyu Song \\ Dongguk University, mksong@dongguk.edu \\ See next page for additional authors
}

Follow this and additional works at: https://digitalcommons.unl.edu/computerelectronicfacpub

Part of the Computer Engineering Commons

Lee, Dongjin; Song, Jaewon; Shin, Jongha; Hwang, Sanghoon; Song, Minkyu; and Wysocki, Tad, "Design of a 1.8V 8-bit 500MSPS Folding-Interpolation CMOS A/D Converter with a Folder Averaging Technique" (2007). Faculty Publications in Computer \& Electronics Engineering (to 2015). 4.

https://digitalcommons.unl.edu/computerelectronicfacpub/4

This Article is brought to you for free and open access by the Electrical \& Computer Engineering, Department of at DigitalCommons@University of Nebraska - Lincoln. It has been accepted for inclusion in Faculty Publications in Computer \& Electronics Engineering (to 2015) by an authorized administrator of DigitalCommons@University of Nebraska - Lincoln. 


\section{Authors}

Dongjin Lee, Jaewon Song, Jongha Shin, Sanghoon Hwang, Minkyu Song, and Tad Wysocki 


\title{
Design of a 1.8V 8-bit 500MSPS Folding-Interpolation CMOS A/D Converter with a Folder Averaging Technique
}

\author{
Dongjin Lee*, Jaewon Song*, Jongha Shin*, Sanghoon Hwang*, Minkyu Song*, and Tad Wysocki** \\ *Dept. of Semiconductor Science, Dongguk University, Seoul, 100-715, Korea \\ ** SECTE, Univ. of Wollongong, Australia \\ E-mail: mksong@,dongguk.edu
}

Abstract - In this paper, a CMOS analog-to-digital converter (ADC) with an 8 -bit $500 \mathrm{MSPS}$ at $1.8 \mathrm{~V}$ is designed. The architecture of the proposed ADC is based on a Folding ADC with a cascaded-folding and a cascaded-interpolation structure. A self-linearized preamplifier with source degeneration technique and a folder averaging technique for the high-performance are introduced. Further, a novel auto-switching encoder is also proposed. The chip has been fabricated with $0.18 \mu \mathrm{m}$ 1-poly 5-metal CMOS technology. The active chip area is $0.79 \mathrm{~mm}^{2}$ and it consumes about $200 \mathrm{~mW}$ at $1.8 \mathrm{~V}$ power supply. The DNL and INL are within $\pm 0.6 / \pm 0.6 \mathrm{LSB}$, respectively. The measured result of SNDR is $47.05 \mathrm{~dB}$.

\section{INTRODUCTION}

The demands for analog-digital interface are now being increased, due to the development of SOC (System-On-a Chip) technique. Specially, a high speed and low power A/D Converters (ADC) are key elements in next-generation communication systems. In the field of high speed ADC, full flash type architecture is known to be the most popular one. However, it consumes large chip area and power dissipation, because of many comparators. In order to overcome the drawbacks of the flash type ADC, folding and interpolation techniques are proposed [1]-[5]. The folding-interpolation type ADC has the advantage of small number of comparators and chip area, while the operating speed is same as that of flash type [2]. For that reason, we propose a low power ADC using folding and interpolation technique for UWB system in this paper.

\section{ARCHITECTURE}

The block diagram of the proposed ADC is shown in Fig. 1. It consists of the pre-processing block, the coarse ADC (3bit), the fine ADC (5-bit), and the digital signal processing block including a digital error correction. With the principle of folding-interpolation architecture, the MSB 3-bit and the LSB 5-bit are divided into a coarse ADC and a fine ADC, respectively. Further, the fine $\mathrm{ADC}$ is composed of the proposed cascaded-folding cascaded-interpolation for a highspeed operation. The pre-processing block is needed to amplify the analog input signal. A proposed self-linearized pre-amplifier with source degeneration technique is adopted for the high-linearity performance in the pre-processing block. Moreover, in the folding block a folding amplifier with averaging folder are described. Also, we introduced a novel auto-switching encoder for the efficient digital processing.

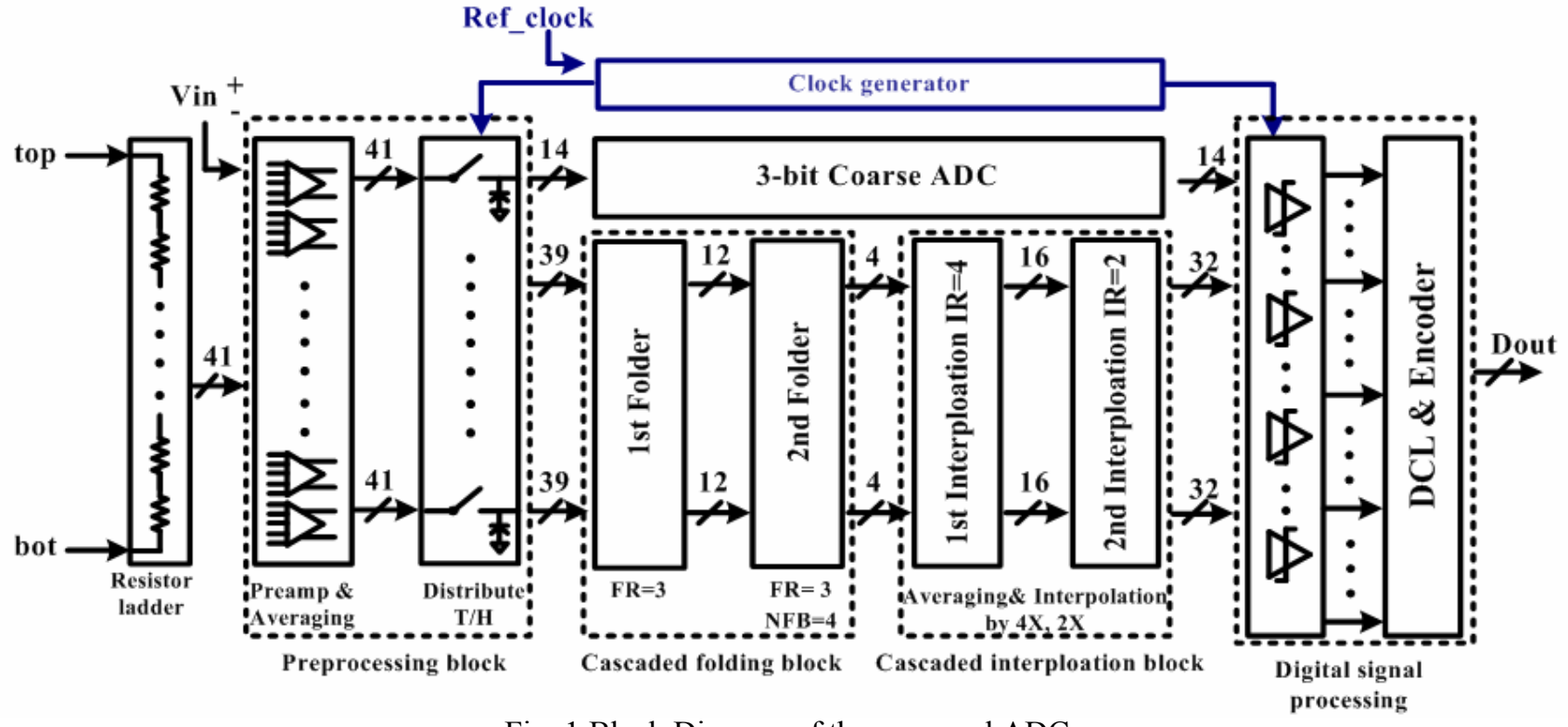

Fig .1 Block Diagram of the proposed ADC 


\section{CIRCUIT DESIGN}

\section{A. Self-Linearized Pre-Amplifier}

The linearity of the pre-amplifier ${ }^{[4]}$ is more important than the voltage gain in the pre-processing block. Therefore, a self-linearized pre-amplifier with the source degeneration technique is proposed for the high-linearity performance. As shown in Fig 2 (a), a degeneration resistor is inserted into the source terminal of input MOSFET. Thus, the linearity of input signal is increased by the degeneration function. Due to the principle of degeneration, the voltage of $R_{S}$ is drop by $I_{D}$, when the $V_{i n}$ is increasing. Since the gate-source overdrive input voltage is protected at the degeneration resistor $R_{s}$, a smoother variation of $I_{D}$ appears at the output node. Fig. 2 (b) shows the input range of the proposed pre-amplifier with $\mathrm{Rs}=0$ and $\mathrm{Rs} \neq 0$.

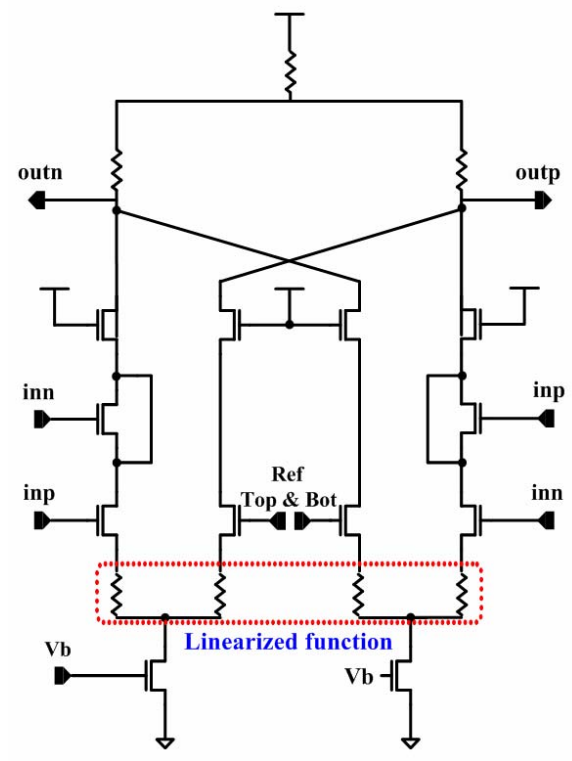

(a)

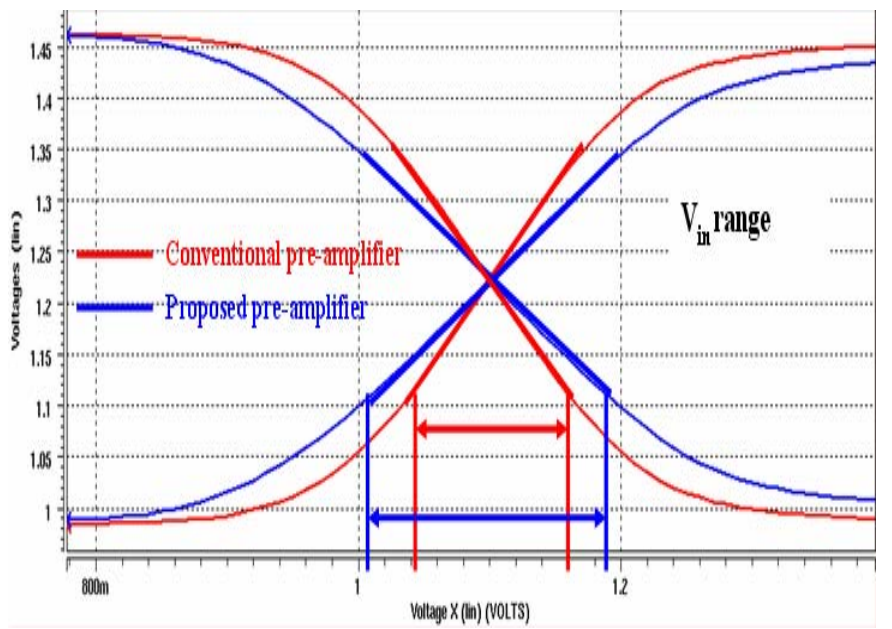

(b)

Fig. 2 Self-Linearized Pre-Amplifier (a) Circuit Diagram (b) Input Range with $R_{S}=0$ and $R_{S} \neq 0$

\section{B. Folder Averaging Technique}

The distribution of the current at the folding blocks is asymmetrical because of the mismatching problems at the folding input transistors. The asymmetric errors cause the non-linearity errors of the folding signals and decrease the performance of ADC. In conventional ones, a dummy folder is inserted to reduce the mismatching problems for the odd number of the folding amplifiers, as shown in Fig. 3 (a). In this paper, to reduce the non-linearity errors, a folding amplifier with a folder averaging is proposed in Fig. 3 (b). The averaging folder is composed of both the last folder and an inserted folder. Further, two folders are connected by common unit current source. Therefore, the size of the proposed averaging folder is half of the other differential pairs. Since the input of the averaging folder is driven by the over range of the pre-processing block, the current of the folding output is averaged with symmetry. Consequently, the zero-crossing error is reduced and the linearity of the folding signal is improved, compared to the conventional ones. As shown in Fig. 4, the asymmetric errors of the proposed folding signals are significantly decreased by the averaging folders.

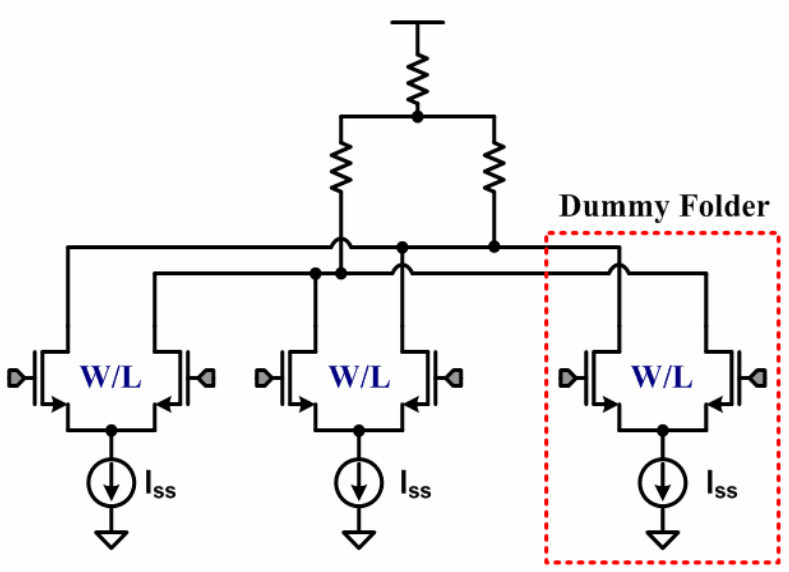

(a)

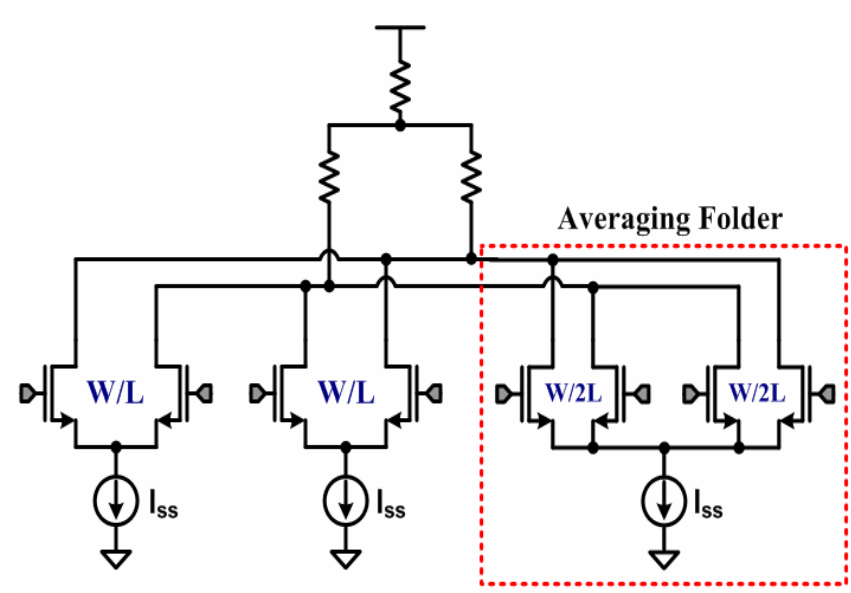

(b)

Fig. 3 Folder Averaging Circuit (a) Conventional Dummy Folder in $\mathrm{FR}=2$ (b) Proposed Folder Averaging in $\mathrm{FR}=2$ 


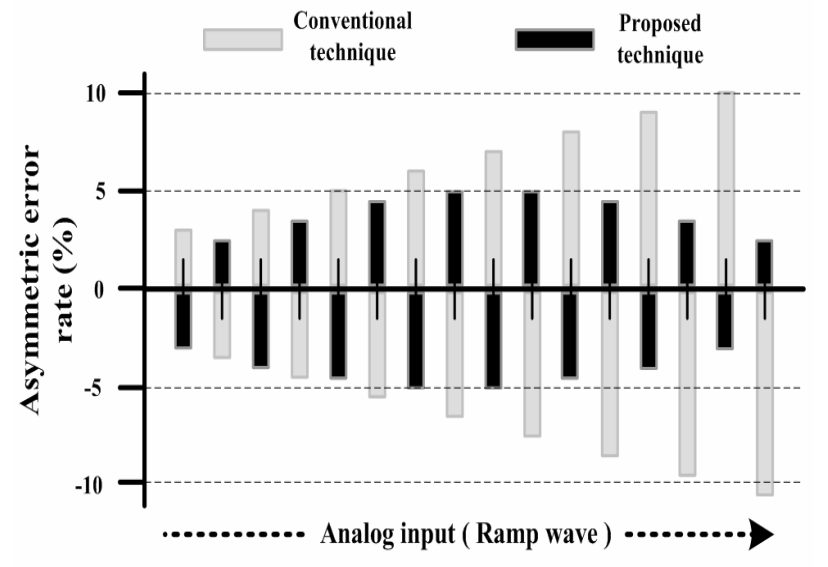

Fig. 4 Asymmetric Error Comparison for Folder Averaging

\section{Auto-Switching Encoder}

Conventionally, the output of the folding block has the reverse-thermometer codes in the folding-interpolation ADC. Thus the digital block of the folding-interpolation ADC must include a switching block for the conversion of the reversethermometer code. However, it is very complicated and hard to implement. In this paper, a novel auto-switching encoder to convert the reverse-thermometer code into the binary code is proposed. The efficient digital algorithm used in the proposed auto-switching encoder is described in Fig. 5. It is a simple example for an algorithm of 3-bit auto-switching encoder. Initially, the thermometer codes, the outputs of comparator, are converted to " 1 " at the boundary of " 0 " and "1" by an XOR gate. As shown in Fig. 6, then, the converted digital code generates the output code through an OR gate. Consequently, the proposed algorithm generates the desired digital output code without any unnecessary switching blocks.

\section{Cascaded-Folding Cascaded-Interpolation Structure}

In general, $3+5$ structure is employed by FR (Folding Rate $)=8, \quad N F B($ Num. of Folding Block $)=4$, and IR (Interpolation Rate) $=8$ in an 8 -bit F/I ADC [1][2]. But if 8 of FR and 8 of IR are chosen, the input signal bandwidth is limited by the folding rate [3], and the output of the folding signal has a large load capacitance by the interpolation rate.
Thermometer \& Reverse thermometer code Input (NO. 1 8)

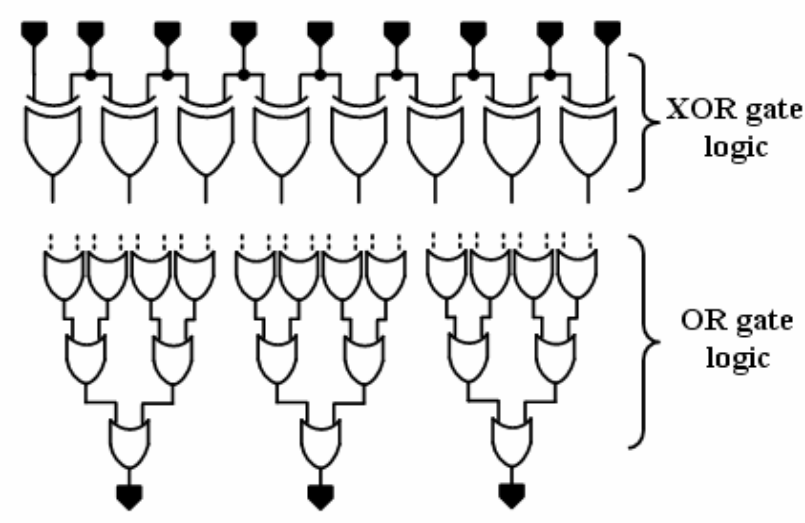

Binary code 3-bit output

Fig. 6 Auto-Switching Encoder

It is a drawback for a high-speed F/I ADC. As a consequence, we proposed cascaded-folding $(\mathrm{FR}=3 \times 3)$ cascaded-interpolation $(\mathrm{IR}=4 \times 2)$ structure. The proposed cascaded-folding cascaded-interpolation structure has a wide input bandwidth and a small load capacitance, compared to the conventional $3+5$ structure $(\mathrm{FR}=8, \mathrm{IR}=8)$. Thus, the proposed cascaded-folding cascaded-interpolation is appropriate for high-speed operation.

\section{EXPERIMENTAL RESULTS}

The proposed folding-interpolation ADC has been fabricated with a $0.18 \mu \mathrm{m} 1$-poly 5-metal CMOS technology. Fig. 7 shows the layout drawing of the proposed ADC. The active chip area is $0.79 \mathrm{~mm}^{2}$. Further, the ADC consumes $200 \mathrm{~mW}$ from $1.8 \mathrm{~V}$ supply when the clock is $500 \mathrm{MHz}$. As shown in Fig. 8 (a), the measured SNDR is $47.05 \mathrm{~dB}$ when the input frequency is $30 \mathrm{MHz}$ at $300 \mathrm{MHz}$ clock frequency. The measured INL and DNL are within 0.6LSB respectively in Fig 8 (b). The experimental results indicate that the proposed folding-interpolation ADC has the desired performance.

\begin{tabular}{|l|l|l|l|l|l|l|l|l|l|}
\hline \multicolumn{1}{|c|}{ Thermometer code (MSB) } & \multicolumn{4}{|c|}{ Encoder } \\
\hline 0 & 0 & 0 & 0 & 0 & 0 & 0 & 0 & 0 & 0 \\
\hline 0 & 0 & 0 & 0 & 0 & 0 & 1 & 0 & 0 & 1 \\
\hline 0 & 0 & 0 & 0 & 0 & 1 & 0 & 0 & 1 & 0 \\
\hline 0 & 0 & 0 & 0 & 1 & 0 & 0 & 0 & 1 & 1 \\
\hline 0 & 0 & 0 & 1 & 0 & 0 & 0 & 1 & 0 & 0 \\
\hline 0 & 0 & 1 & 0 & 0 & 0 & 0 & 1 & 0 & 1 \\
\hline 0 & 1 & 0 & 0 & 0 & 0 & 0 & 1 & 1 & 0 \\
\hline 1 & 0 & 0 & 0 & 0 & 0 & 0 & 1 & 1 & 1 \\
\hline
\end{tabular}

\begin{tabular}{|l|l|l|l|l|l|l|l|l|l|}
\hline \multicolumn{7}{|c|}{ Thermometer code } & \multicolumn{3}{|c|}{ Encoder } \\
\hline 0 & 0 & 0 & 0 & 0 & 0 & 0 & 0 & 0 & 0 \\
\hline 0 & 0 & 0 & 0 & 0 & 0 & 1 & 0 & 0 & 1 \\
\hline 0 & 0 & 0 & 0 & 0 & 1 & 0 & 0 & 1 & 0 \\
\hline 0 & 0 & 0 & 0 & 1 & 0 & 0 & 0 & 1 & 1 \\
\hline 0 & 0 & 0 & 1 & 0 & 0 & 0 & 1 & 0 & 0 \\
\hline 0 & 0 & 1 & 0 & 0 & 0 & 0 & 1 & 0 & 1 \\
\hline 0 & 1 & 0 & 0 & 0 & 0 & 0 & 1 & 1 & 0 \\
\hline 1 & 0 & 0 & 0 & 0 & 0 & 0 & 1 & 1 & 1 \\
\hline
\end{tabular}

\begin{tabular}{|l|l|l|l|l|l|l|l|l|l|}
\hline \multicolumn{1}{|c|}{ Thermometer code (LSB) } & \multicolumn{3}{|c|}{ Encoder } \\
\hline 0 & 0 & 0 & 0 & 0 & 0 & 0 & 0 & 0 & 0 \\
\hline 0 & 0 & 0 & 0 & 0 & 0 & 1 & 0 & 0 & 1 \\
\hline 0 & 0 & 0 & 0 & 0 & 1 & 0 & 0 & 1 & 0 \\
\hline 0 & 0 & 0 & 0 & 1 & 0 & 0 & 0 & 1 & 1 \\
\hline 0 & 0 & 0 & 1 & 0 & 0 & 0 & 1 & 0 & 0 \\
\hline 0 & 0 & 1 & 0 & 0 & 0 & 0 & 1 & 0 & 1 \\
\hline 0 & 1 & 0 & 0 & 0 & 0 & 0 & 1 & 1 & 0 \\
\hline 1 & 0 & 0 & 0 & 0 & 0 & 0 & 1 & 1 & 1 \\
\hline
\end{tabular}

Fig. 5 The Proposed Algorithm Converted the Thermometer Codes into the Binary Codes 


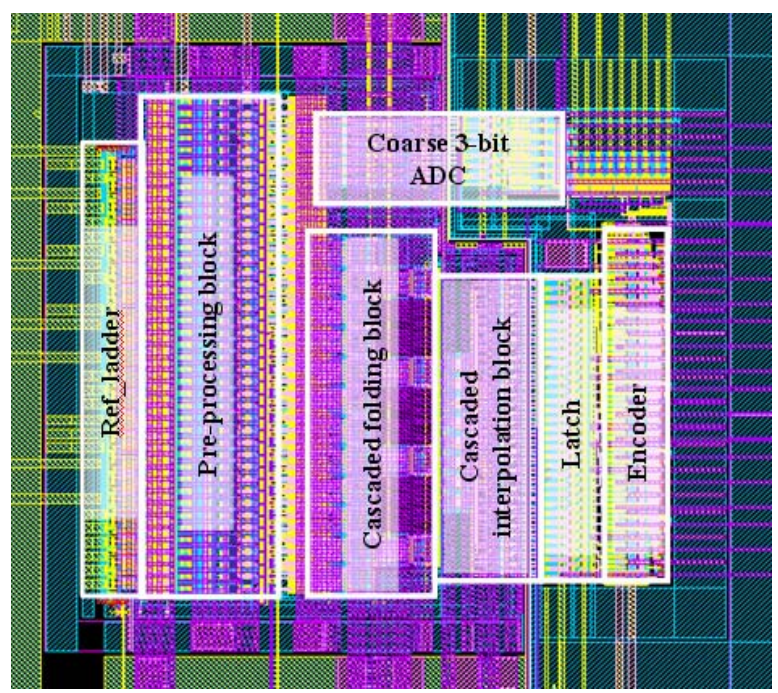

Fig. 7 Chip Layout Drawing of the proposed ADC

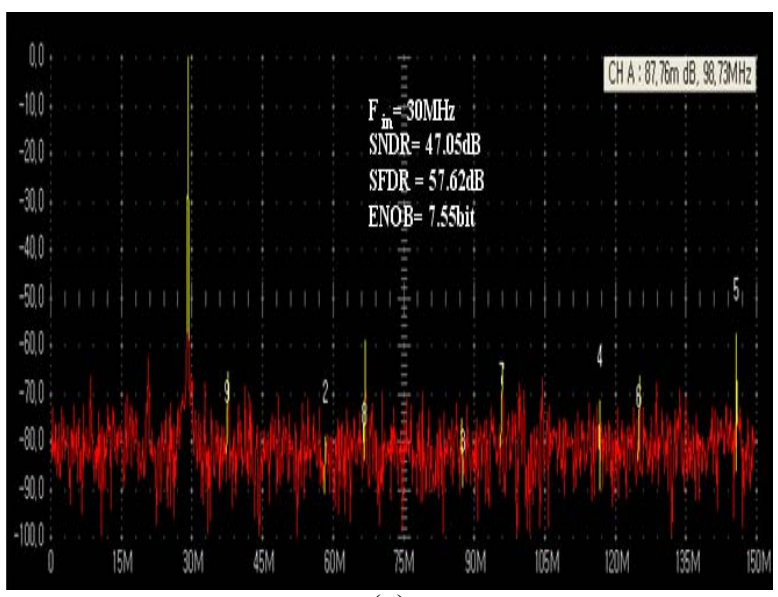

(a)
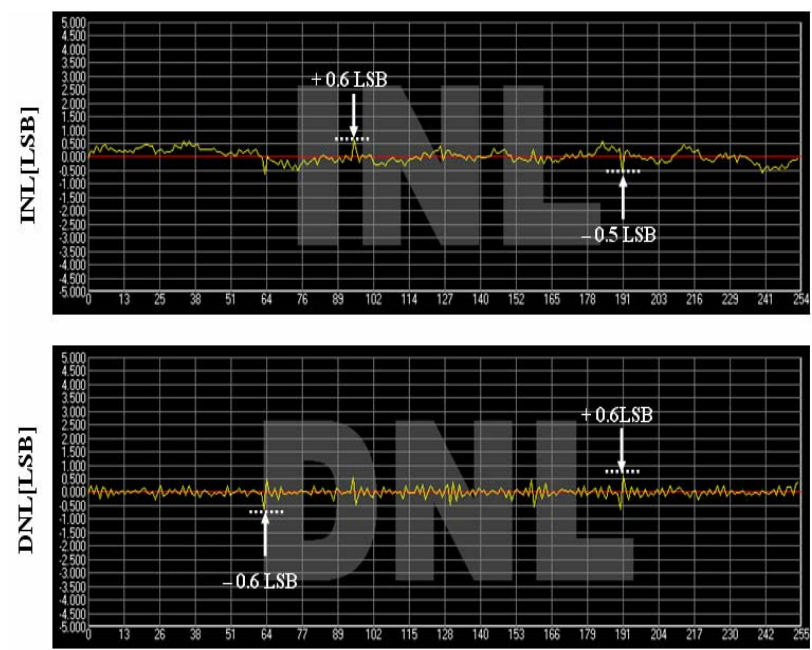

(b)

Fig 8. Measured Results

(a) Measured FFT (b) Measured INL\&DNL

\section{CONCLUSION}

A 1.8V 8-bit 500MSPS CMOS ADC has been described. The architecture of the proposed ADC was based on a folding ADC using a cascaded-folding cascaded-interpolation structure. The self-linearized pre-amplifier with source degeneration technique, the folder averaging technique, and an auto-switching encoder were discussed for the desired performance of the ADC. The effective chip area was $0.79 \mathrm{~mm}^{2}$, the ADC consumed $200 \mathrm{~mW}$ at $1.8 \mathrm{~V}$ supply. The measured $\mathrm{ENOB}$ of the proposed $\mathrm{ADC}$ was 7.55 bits when the input frequency was $30 \mathrm{MHz}$ at $500 \mathrm{MSPS}$. The measured results of the ADC are summarized in Table 1.

Table 1. Measured Results of the proposed ADC

\begin{tabular}{|c|c|}
\hline Resolution & 8-bit \\
\hline Sampling Freq. & 500MSPS \\
\hline Power Supply & $1.8 \mathrm{~V}$ (Analog + Digital) \\
\hline Analog Input Range & Diff. 1Vpp \\
\hline $\begin{array}{c}\text { DNL / INL } \\
\text { (Measured Data) }\end{array}$ & $\pm 0.6 \mathrm{LSB} / \pm 0.6 \mathrm{LSB}$ \\
\hline $\begin{array}{c}\text { ENOB } \\
\text { (Measured Data) }\end{array}$ & $7.55 \mathrm{bits}(\mathrm{Fs}=300 \mathrm{MHz})$ \\
\hline Power & $200 \mathrm{~mW}$ \\
\hline Core Area & $920 \mathrm{um} \times 860 \mathrm{um}$ \\
\hline Process & Samsung $0.18 \mathrm{um}$ CMOS \\
\hline
\end{tabular}

\section{ACKNOWLEDGEMENT}

This work was partly sponsored by ETRI SOC Industry Promotion Center, Human Resource Development Project for IT SOC Architect. The authors are thankful for the support of IC Design Education Center (IDEC), KAIST, Korea.

\section{REFERNCES}

[1] R.Grift. I. Rutten and M. Veen, "An 8-bit Video ADC Incorporation Folding and Interpolation Technique," IEEE J. Solid-State Circuits, vol. SC-22, no. 6, pp. 994-953. Dec. 1987.

[2] Pieter VorenKamp. "A 12-b 60-MSample/s Cascaded Folding and Interpolation ADC," IEEE J. Solid-State Circuits, vol. 32. 12 1876-1886. Dec. 1997.

[3] Evandro Mazina Martinx and Elnatan Chagas Ferreira, "An 8-bit Folding A/D Converter with a New Interpolation Technique," Analog Intergrated Circuits and Signal Processing, vol 41, pp 237-252, 2004.

[4] Zheng-Yu Wang, Hui Pan, Chung-Ming Chang, HaiRong Yu and M. Frank Chang. "A 600MSPS 8-bit Folding ADC in 0.18um CMOS" 5 SOVC, pp. 424-427, June. 2004.

[5] M.P. Flynn, B. Sheahan, "A 400-msample/s, 6-b cmos folding and interpolating adc", IEEE JSSC, Vol. 33, pp. 1932-1938, Dec. 1998. 